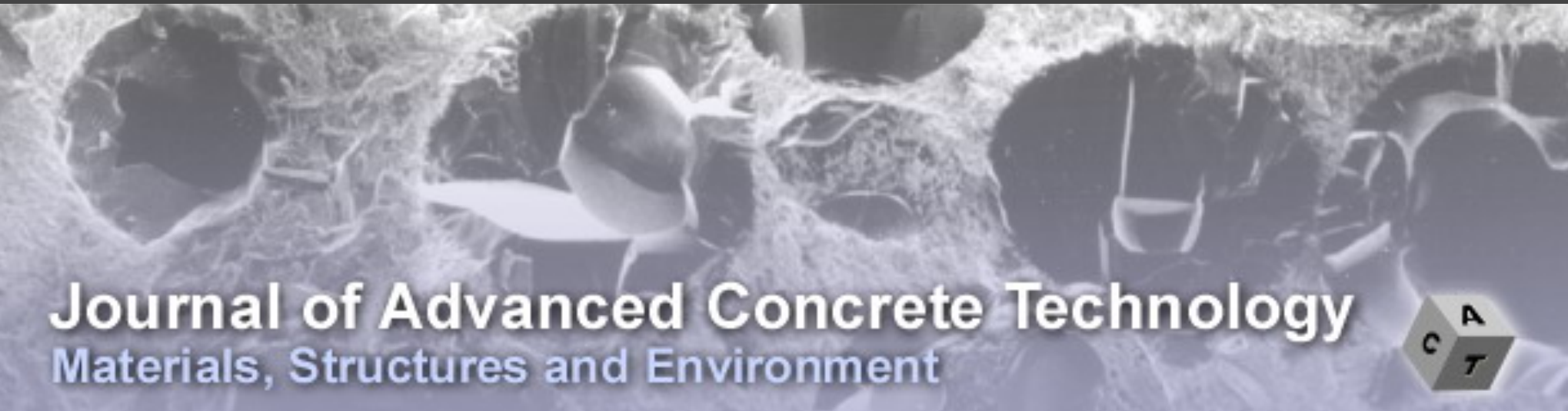

\title{
Ultrasonic nondestructive testing of cement grouting quality in corrugated pipes based on impact-echo
}

Qingbang Han, Jin Cheng, Honghui Fan, Cheng Yin, Changping Zhu

Journal of Advanced Concrete Technology, volume 12 (2014 ), pp. 503-509

\section{Related Papers Click to Download full PDF!}

Nondestructive Evaluation of Damaged Concrete due to Freezing and Thawing by Elastic-Wave Method Masayasu Ohtsu

Journal of Advanced Concrete Technology, volume 3 (2005), pp. 333-341

Nondestructive evaluation of setting and hardening of cement paste based on ultrasonic propagation characteristics

Toshiro Kamada, Shinya Uchida, Keitetsu Rokugo

Journal of Advanced Concrete Technology, volume 3 (2005), pp. 343-353

Ultrasonic Wave Reflection Approach to Evaluation of Fresh Concrete Friction

Yannick Vanhove, Chafika Djelal, Thierry Chartier

Journal of Advanced Concrete Technology, volume 6 (2008), pp. 253-260

Kinematics of Corrosion Damage Monitored by Acoustic Emission Techniques and Based on a Phenomenological Model

Yuma Kawasaki, Tomoe Kobarai, Masayasu Ohtsu

Journal of Advanced Concrete Technology, volume 10 (2012), pp. 160-169 


\title{
Ultrasonic Nondestructive Testing of Cement Grouting Quality in Corrugated Pipes Based on Impact-echo
}

\author{
Qingbang Han $^{1}$, Jin Cheng ${ }^{2}$, Honghui Fan ${ }^{3}$, Cheng Yin ${ }^{4}$ and Changping Zhu ${ }^{5}$
}

\begin{abstract}
This research aims to detect cement grouting defects in corrugated pipes using the ultrasonic impact-echo method. A combined signal processing technique that included de-convolution technique, information entropy and cross-correlation were proposed to identify and characterize defects. Several numerical models were constructed to simulate and analyze the results using the finite element method. The stripping size of the defects in the corrugated pipe was recognized by analyzing the information entropy and correlation coefficients of the echo signals. Experiments were carried out on a concrete specimen containing artificial defects with varying stripping sizes and numbers of elements. The experimental results were consistent with the simulated predictions.
\end{abstract}

\section{Introduction}

Unsatisfactory corrugated pipe grouting quality in bridges can lead to serious accidents. Corrugated pipes are widely used as reserved channels in post-tensioned, pre-stressed beams. The steel strands are the key load-carrying components in corrugated pipes. With high strength and low relaxation, steel strands exert pre-stress to strengthen concrete structures, such as high-rise buildings and suspension bridges (Beben 2011). The cement grouting is one of the most critical processes. Poor corrugated pipe grouting quality will result in malfunction of the steel strands and safety accidents.

Some researchers have developed a method to detect inner defects using ultrasonic guided waves to monitor the propagation velocity, stress levels and energy intensity variations in pre-stressed steel strands (Delvasto et al. 2010; Chaki and Bourse 2009). However, ultrasonic guided wave methods is relative suitable for poor quality of grouting, which can cause more difference, besides, it is unable to provide the exact position or size of the grouting defects. The ultrasonic pulse-echo method is widely used in ultrasonic nondestructive tests in concrete construction (Liang and $\mathrm{Su} 2001$ ). A method based on empirical mode decomposition using the Hilbert Huang

${ }^{1}$ Professor, College of IOT Engineering, Hohai university Changzhou, China.

*Corresponding author, E-mail: Hqb0092@163.com

${ }^{2}$ Master, College of IOT Engineering, Hohai university, Changzhou, China.

${ }^{3}$ Doctor, School of Computer Engineering, Jiangsu University of Technology, Changzhou, China.

${ }^{4}$ Doctor, College of IOT Engineering, Hohai university, Changzhou, China.

${ }^{5}$ Professor, College of IOT Engineering, Hohai university Changzhou, China. transform was proposed to decompose the echo signals into internal model functions. Fourier transform was then used to analyze the functions in the time and frequency domains (Lin et al. 2009). Although many information process methods have been successfully applied to test concrete structures, very few provide satisfactory data for assessing the internal defects in corrugated pipes because the weak signals reflected by grouting defects are generally impossible to discern in the background due to the strong scattering from the concrete. In this paper, a combination signal processing technique using the information entropy and cross-correlation was proposed for identifying grouting quality.

\section{Problem presentation}

The post-tensioned concrete structure is generally composed of four parts: the concrete block, in which the deformed steel bars are usually poured to improve strength; the corrugated pipe, which is made up of metal or plastomer and used as a reserve channel; the cement paste, which is used to consolidate the grouting; , and the steel strands, which can improve the lifespan of the concrete structures. Figure 1 displays the configuration of the concrete structure with a corrugated pipe. The

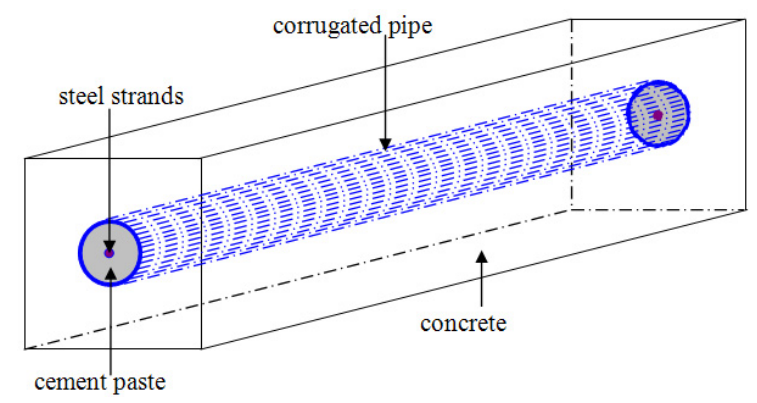

Fig. 1 Configuration of the concrete structure containing the corrugated pipe. 
corrugated pipe is located in the middle and the steel strands are fixed inside the corrugated pipe. After the steel strands are pre-stressed, the cement paste is grouted into the corrugated pipe to provide support force for the steel strands. This structure is rather complicated. To collect impact-echo signals, the acoustic wave should traverse the concrete block and the corrugated pipe before reflecting along the same path after encountering the un- grouted defect. The desired signals are rather weak because of the strong scattering of the concrete and the pipe. Thus, identifying these signals and excluding their effects is vital to the problem.

\section{Finite element simulation}

\subsection{Numerical modeling}

To avoid long time calculations of 3D model, the contractible 2D model with proper boundary conditions instead is designed as can be seen in Fig. 2. It shows the top view of the $3 \mathrm{D}$ model. The model is a $140 \mathrm{~cm} \times 30 \mathrm{~cm}$ rectangle, with an external diameter of the corrugated pipe of $9 \mathrm{~cm}$ and a thickness of $0.3 \mathrm{~cm}$. The diameter of a steel strand is $1 \mathrm{~cm}$. The model is divided into 7 sections from $\mathrm{P} 0$ to $\mathrm{P} 6$. The excitation and receiving location are on the same side of each section to receive the echo signal. The distance between the transducer and the receiver is $5 \mathrm{~cm}$. The diameter of transducer is $1 \mathrm{~cm}$, and the receive signals can be collected on some points location directly. Different sizes of defects in the corrugated pipes were designed. The detailed sizes are listed in Table 1. The parameters of the medium used in the simulation are shown in Table 2. The time step is $6 \times 10^{-7} \mathrm{~s}$, and the mesh size is $0.05 \mathrm{~cm}$. The excitation signal is modulated by the Hanning function according to

$$
S(t)=\frac{1}{2}\left(1-\cos \left(2 \pi t / t_{0}\right)\right) * \cos \left(2 \pi f\left(t-\frac{t_{0}}{2}\right)\right) *\left(t<t_{0}\right)
$$

Where, $\mathrm{f}$ represents excitation frequency and $\mathrm{t} 0$ represents pulse width. $\mathrm{f}=200 \mathrm{kHz}$ and $\mathrm{t} 0=10 \mu \mathrm{s}$.

In Table 2, the steel AISI 4340 is a kind of high-strength and low-alloy steel and all simulation are
Table 1 Sizes of the defects.

\begin{tabular}{ccccccc}
\hline $\begin{array}{c}\text { Size of } \\
\text { defect }\end{array}$ & P1 & P2 & P3 & P4 & P5 & P6 \\
\hline Size in X & $0.03 \mathrm{~cm}$ & $0.06 \mathrm{~cm}$ & $3 \mathrm{~cm}$ & $6 \mathrm{~cm}$ & $9 \mathrm{~cm}$ & $12 \mathrm{~cm}$ \\
Size in Y & $0.02 \mathrm{~cm}$ & $0.04 \mathrm{~cm}$ & $2 \mathrm{~cm}$ & $2 \mathrm{~cm}$ & $2 \mathrm{~cm}$ & $2 \mathrm{~cm}$ \\
\hline
\end{tabular}

Table 2 Parameters used for the medium in the simulation.

\begin{tabular}{ccccc}
\hline Physics & Density & $\begin{array}{c}\text { Young's } \\
\text { modulus }\end{array}$ & $\begin{array}{c}\text { Poisson } \\
\text { ratio }\end{array}$ & $\begin{array}{c}\text { Sound } \\
\text { velocity }\end{array}$ \\
\hline $\begin{array}{c}\text { Concrete } \\
\text { Steel AISI } \\
4340\end{array}$ & $2300 \mathrm{~kg} / \mathrm{m}^{3}$ & $25 \times 10^{9}[\mathrm{pa}]$ & 0.33 & -- \\
$\begin{array}{c}\text { Cement } \\
\text { paste } \\
\text { Air }\end{array}$ & $1980 \mathrm{~kg} / \mathrm{m}^{3}$ & $205 \times 10^{9}[\mathrm{pa}]$ & 0.28 & -- \\
& $1.25 \mathrm{~kg} / \mathrm{m}^{3}$ & $11.6 \times 10^{9}[\mathrm{pa}]$ & 0.25 & -- \\
\hline
\end{tabular}

performed by comsol multiphysics

\subsection{Analysis of the pulse-echo signal}

Figure 3 shows the characteristics of the impact-echo signals. The transient response of a signal for P0 is presented in Fig. 3(a). Considering that the excitation and receiving positions are located on the same surface, the first three waves should correspond to the head wave (lateral wave), the shear wave and the surface wave (Rayleigh wave), which have nothing to do with the research problem. The later waves are the reflected $\mathrm{P}$-waves and $\mathrm{S}$-waves from the corrugated pipe, the defects, the concrete boundary and other structures.

The grouting information is hidden in the $60 \mu \mathrm{s}$ to $160 \mu$ s time range, corresponding to the area from the front wall of corrugated pipe to the bottom of concrete structure. To avoid information loss, the time of signal extends to $200 \mu$ s. However, it is difficult to judge which waves correspond to defect reflections. The truncated transient response in seven sections (from P0 to P6) with an amplitude offset used to elide the $0-60 \mu$ s wave form (the first three waves) is shown in Fig. 3 (b). These waveforms are very similar to each other and difficult to separate from P0 to P6. In the frequency domain shown

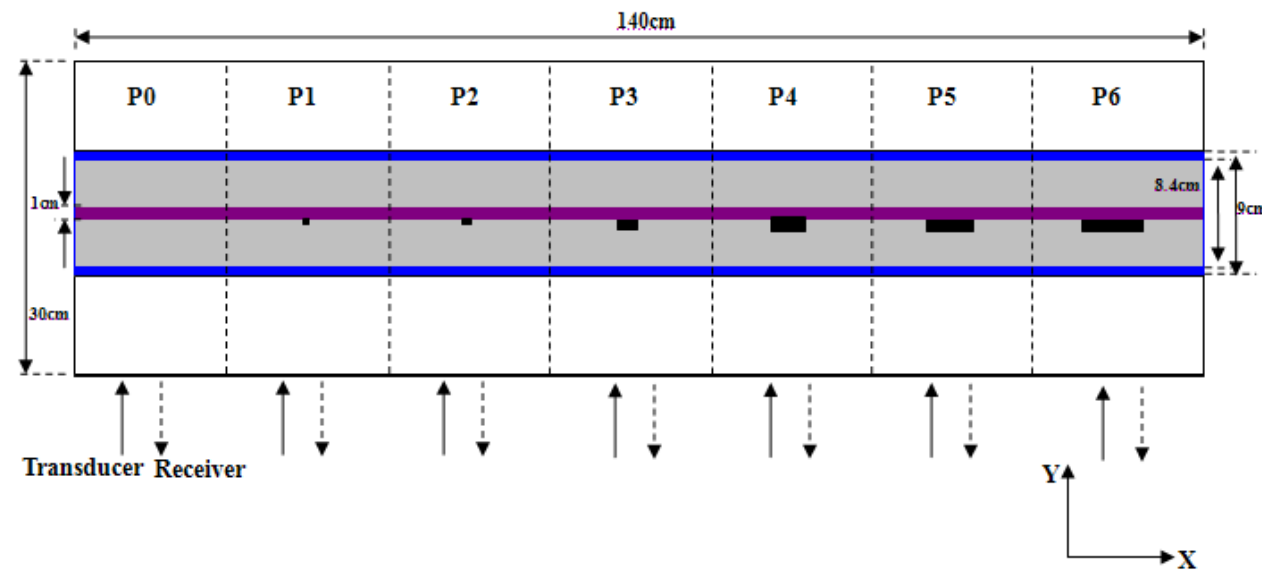

Fig. 2 Simulation 2D model of the concrete and corrugated pipe. 


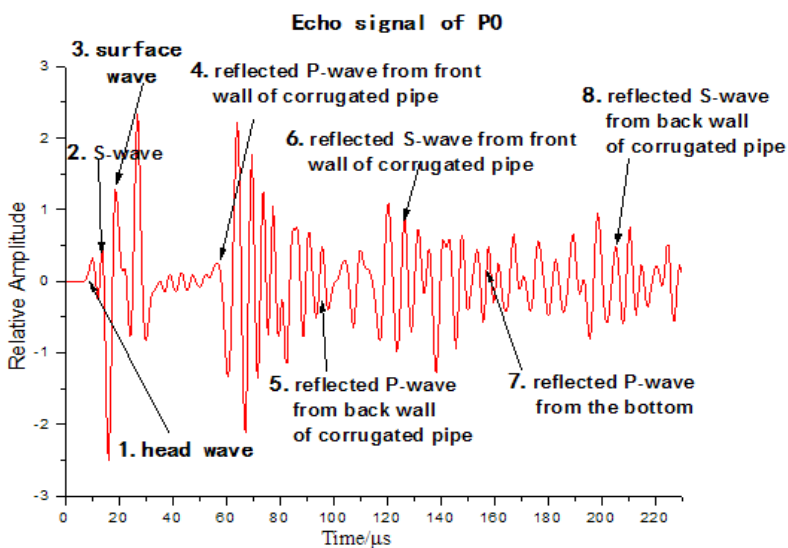

(a) Transient response of an echo signal in the $\mathrm{P}_{0}$ section.

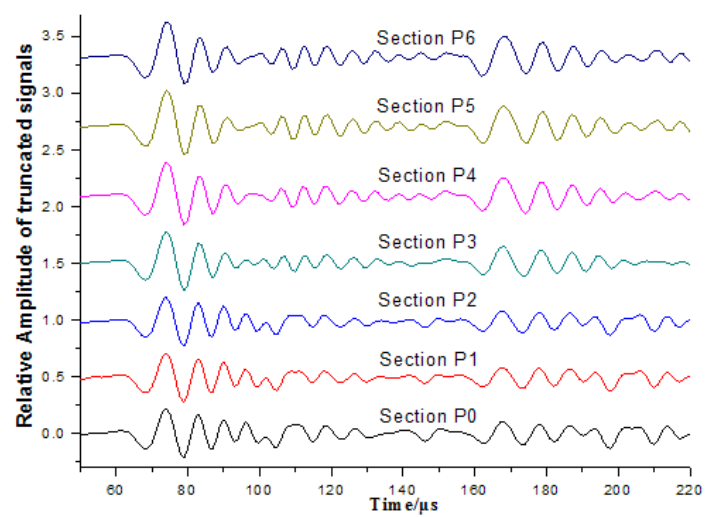

(b) Comparison of echo signals for sections P0 to P6.

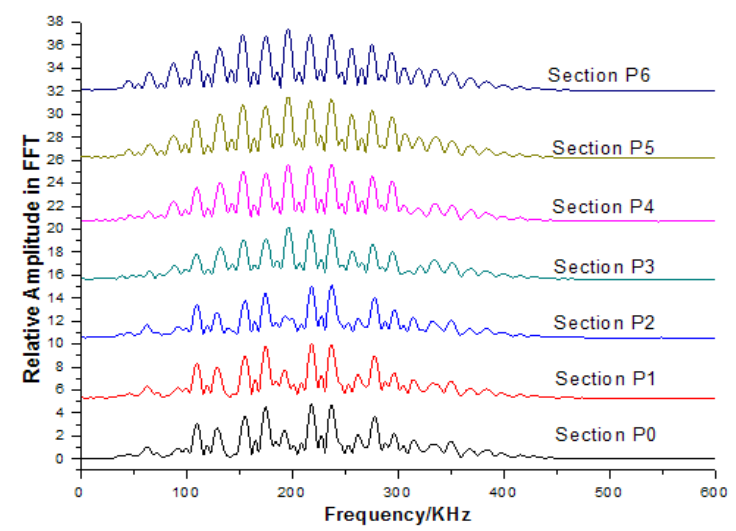

(c) Comparison of frequency spectrum for sections P0 to P6.

Fig. 3 Characteristics of the echo signals.

in Fig. 3 (c), it is also difficult to discern differences among the waveforms. Compared with the stronger scattering of corrugated pipes (particularly for metal pipes), the expected signals caused by the defect are very weak. The key problem is how to exclude the influence of the corrugated pipe and obtain useful information pertaining to the defects.

\subsection{Procedures}

\subsubsection{Technology procedure}

The echo signals containing internal information from the specimen can be collected by a receiver after the transducer transmits the ultrasonic signal. Each echo signal includes a wealth of internal information of the concrete structure. We must isolate the information that we need to distinguish the defects. There are differences in the system signals between structure with no defects and structures containing defects. We can treat the concrete specimen as a system.

The flowchart for the technology procedures for echo signal processing is shown in Fig. 4. The truncated echo signal $Y(t)$ (excluding the surface waves) is processed by excitation signal $S(t)$, which can be used for de-convolution with $Y(t)$ to achieve the system signal $N(t)$. Then, the processes of information entropy and cross-correlation are applied to describe the grouting quality.

\subsubsection{System signal}

Treating the test concrete specimen as a system, the echo signal $Y(t)$ can be expressed as

$$
Y(t)=S(t) * N(t)
$$

where $S(t)$ is the excitation signal, "* " represents the convolution operation and $N(t)$ indicates the system signal that refers to a signal that excludes the excitation effect. The system signal $N(t)$ can then be obtained by de-convolution (Lavrentyev and Beals 2000).

In the actual measurements, excitation signal is defined as $S(t)$, the system signal $N(t)$ can be obtained using

$$
N(t)=Y(t) \otimes S(t)
$$

where, “ $\otimes "$ means de-convolution operation.

\subsubsection{Process of wavelet entropy}

The entropy $H(X)$ of a single discrete random variable $X$ is a measure of its average uncertainty. In statistical mechanics, entropy is essentially a measure of the number of ways in which a system may be arranged, often taken to be a measure of "disorder" (the higher the entropy, the higher the disorder). The overall degree of complexity of a signal is evaluated by considering the entropy values, and entropy also can indicate the instability of system (Perez-Canales et al. 2011; Jaynes 1982; Biatynicki-Birula and Mycielski 1975). Regarding the "no-defect" area as a homogeneous (not concentrated or disorderly) system, that also means disorder. And in the "defects" area, the homogeneity will be interfered so that the "defects" system will represent certain regularity (concentrated or orderly). The more the system state is concentrated (ordered), the lower the information entropy value will be (Biatynicki-Birula 1975). Information entropy can be used to reveal a system's disorder, instability, uncertainty and imbalance. Accordingly, an existing defect in a corrugated pipe in a concrete structure will cause a change in the information entropy of the system.

The mathematical definition of information entropy is 


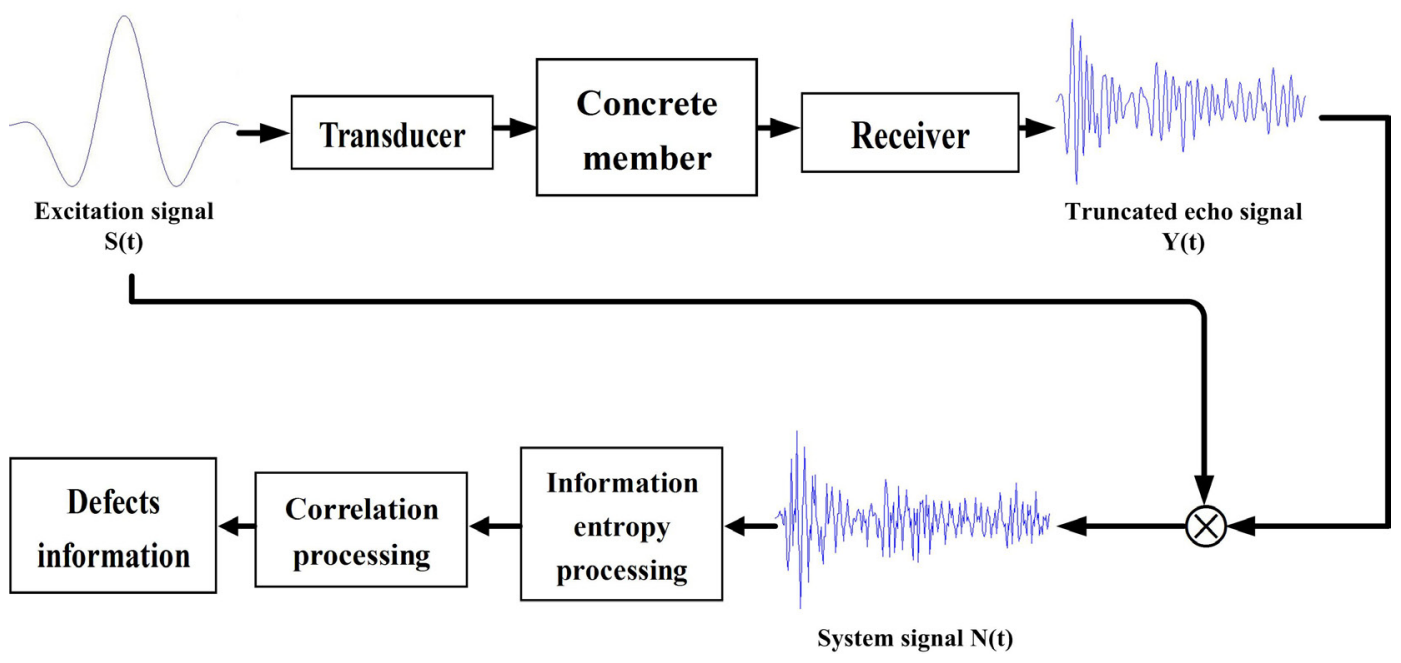

Fig. 4 Flowchart for the technology procedure.

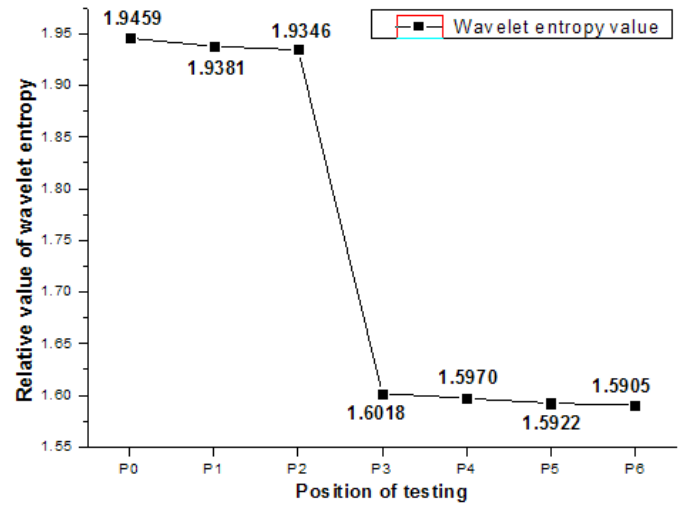

Fig. 5 The relationship between wavelet entropy and the defects.

$$
H(X)=\sum_{n}-P(i) \log P(i), i=1,2, \cdots n
$$

where $X=\left\{x_{1}, x_{2}, \cdots x_{n}\right\}$ is a discrete sample sequence with $\mathrm{n}$ states and $P=\left\{p_{1}, p_{2}, \cdots p_{n}\right\}$ indicates the probability of occurrence of each state (Shannon 1948).

In the wavelet spectrum, $P_{i}$ is the probability of each component energy value of the last grade, which is obtained by multi-resolution analysis $(M R A)$. Assuming that $E_{\text {whole }}$ is the total energy of the wavelet spectrum, we have

$$
\begin{aligned}
& E_{\text {whole }}=E_{A}+\sum E_{D_{i}}, i=1,2 \cdots J \\
& P_{i}= \begin{cases}\frac{E_{A}}{E_{\text {whole }}} \quad, i=J ; \\
\frac{E_{D_{i}}}{E_{\text {whole }}}, i=1,2 \cdots J ;\end{cases}
\end{aligned}
$$

where $E_{D_{1}}$ presents the energy of each $D_{i}$ and $E_{A}$ indicates the energy of $A_{J}$.

The system signal in the simulation is processed using the wavelet entropy, as shown in Fig. 5. The wavelet entropy is largest when there are no defects. It decreases as a function of the defect size, which implies that defect size is related to uncertainty in the energy of the echo signal in the information spectrum. Greater differences in defect size lead to greater differences in wavelet entropy. Although wavelet entropy can provide some information about the defects, there is still much uncertain to the difference of detects. Therefore, it should be combined with another method to judge defects more accurately. In this study, we also used the cross-correlation method.

\subsubsection{Process of cross-correlation}

In the field of signal processing, cross-correlation is used to represent the similarity between two signals. Typically, it is used to detect features in an unknown signal by comparison with a known signal. When inspecting grouting quality, the echo signal collected from the area with no defects is considered the reference signal. That is, the system signal of this area $\left(N_{\text {ref }}(t)\right)$ also acts as a systemic reference signal $(R E F)$. The cross-correlation function represents the degree of correlation between signal $N_{\text {ref }}(t)$ and signal $N_{i}(t)$, which is considered the system signal of a certain area at an arbitrary time $t$ according to

$$
C_{12}(\tau)=\lim _{T \rightarrow \infty} \frac{1}{T} \int_{0}^{T} N_{r e f}(t) N_{i}(t+\tau) d t
$$

The normalized cross-correlation coefficient is

$$
\rho_{i}(\tau)=\frac{C_{i}(\tau)}{\sqrt{C_{\mathrm{ref}}(0) C_{i}(0)}} \leq 1
$$


and $C_{i}(0)$ corresponds to $N_{i}(t)$ (Laasri et al, 2014; Wan and $\mathrm{Wu} 2013)$.

Through the cross-correlation process, the influence of the corrugated pipe and the concrete block can be excluded. Any difference may give an expression of the defects. If the system signal of the $P 0$ section is chosen as the reference signal, the cross-correlation coefficient between it and the other signals can be plotted as shown in Fig. 6 (a). The cross-correlation coefficient decreased as a function of defect size, i.e., a greater coefficient indicates a larger defect. This can be used to identify defects and their relative sizes. The results from the signals from $P 1$ or $P 2$ (two relative small defects), which were used as references, are shown in Fig. 6 (b). The cross-correlation coefficients for $P 0, P 1$ and $P 2$ are very close to one another (Proximity-1), and their other characteristics are similar to Fig. 6(a), implying that selecting any one echo signal using a small defect as a reference signal $N_{\text {ref }}(t)$ does not influence the judgment, which is advantageous for actual testing.

However, in the actual measurement, we do not know which signals correspond to the no defect areas. The information entropy, however, provides a way to judge. Using the signal of the maximum value of the information entropy as a reference, the signal is either from the no-defect area or an area close to a perfect section.

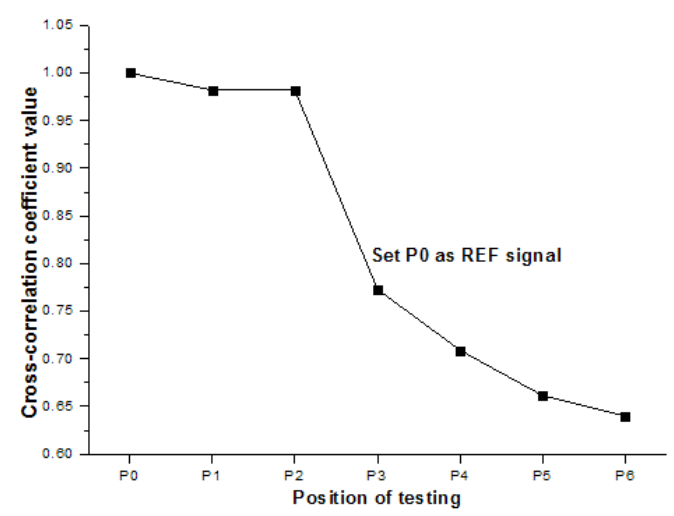

(a) Selecting $P 0$ as REF

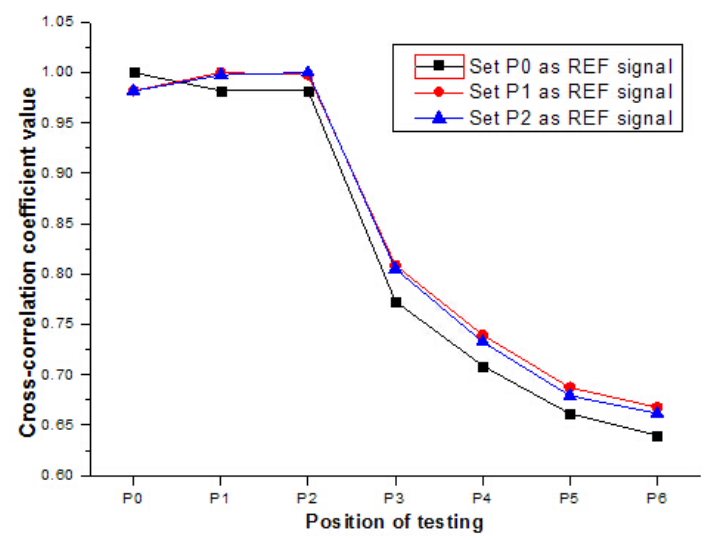

(b) Selecting $P 0, P 1$ and $P 2$ as $R E F$, respectively

Fig. 6 The relationship between the cross-correlation coefficient and the size of defects.
In conclusion, for an actual testing process, all of the echo signals are first processed by de-convolution method to obtain the system signal. Through information entropy analysis, the signal with the maximum value is then chosen as the reference signal. Then, the cross-correlation coefficient is measured to identify the size difference of the defect. Although the accurate defects sizes are still not known, the combined method has the ability to provide an effective path for evaluating the grouting quality.

\section{Experiment}

\subsection{Actual model}

To verify the results obtained from the numerical analyses, experimental studies using an actual model were performed. The corrugated pipe concrete specimen had a size of $2.6 \mathrm{~m} \times 0.5 \mathrm{~m} \times 1 \mathrm{~m}$ and contained an internal corrugated pipe with an inner diameter of $69 \mathrm{~mm}$, a wall thickness of $3 \mathrm{~mm}$ and a length of $2.6 \mathrm{~m}$, as shown in Fig. 7. Figure 7 also shows the top view of the actual model. Four different types of defects were designed in the specimen. Their detailed sizes are listed in Table 3.

The echo signals were collected from positions A to F. A high-power pulser that we designed, with $200 \mathrm{kHz}$ and $200 \mathrm{~kW}$, was used to excite ultrasound through the transducer. The distance between the transducers was 5 $\mathrm{cm}$.

\subsection{Analysis and results}

A typical echo signal collected during actual testing is shown in Fig. 8. A distinct transient response was observed, which was similar to the simulated response shown in Fig. 8(a). Several obvious reflected waves were clearly identified. High-frequency signals $(>250 \mathrm{kHz})$ were absorbed for strong scattering, according to Fig. 8(b).

Table3 Size of the defects in the actual testing.

\begin{tabular}{ccccc}
\hline Size of Defect & B & C & D & E \\
\hline Size in X & $5 \mathrm{~cm}$ & $10 \mathrm{~cm}$ & $15 \mathrm{~cm}$ & $20 \mathrm{~cm}$ \\
Size in Y & $4.14 \mathrm{~cm}$ & $4.14 \mathrm{~cm}$ & $4.14 \mathrm{~cm}$ & $4.14 \mathrm{~cm}$
\end{tabular}

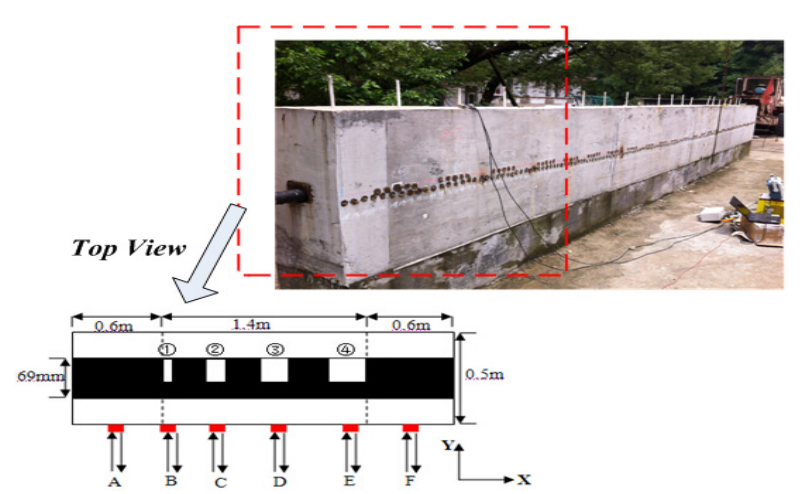

Fig.7 The actual concrete specimen containing some artificial defects in the corrugated pipe. 


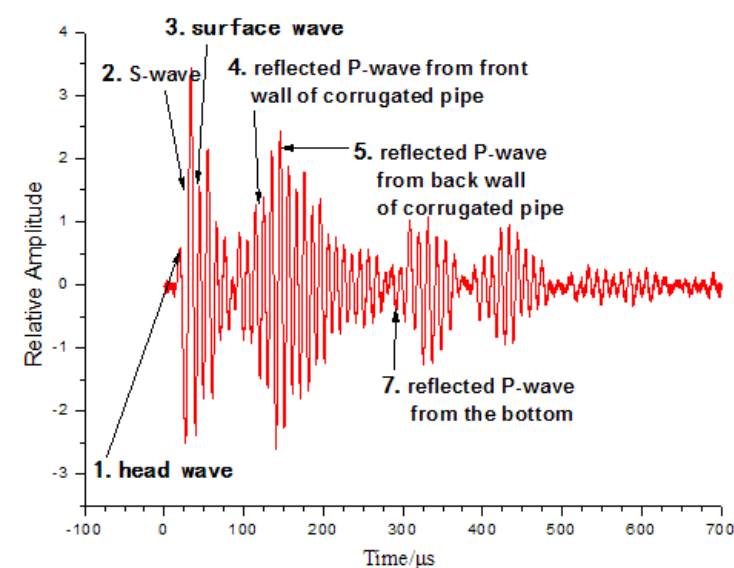

(a) Transient response

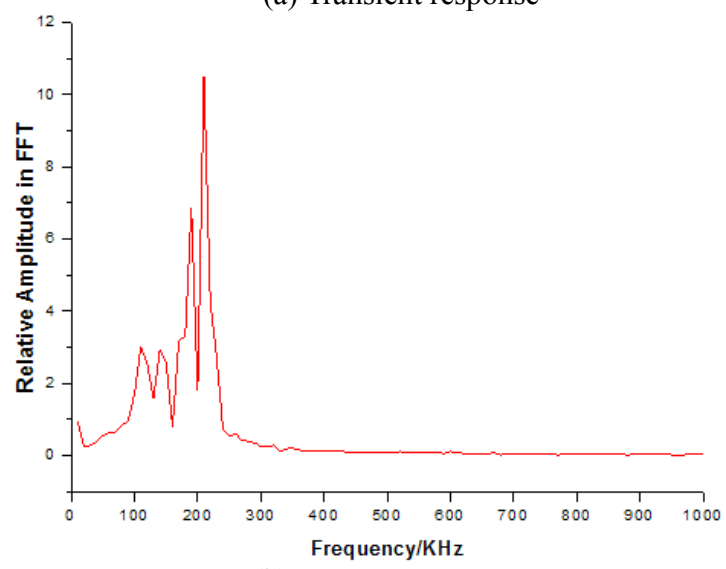

(b) Frequency spectrum

Fig. 8 The echo signal collected during actual testing.

After collecting excited signal by oscilloscope, using the wavelet entropy technology mentioned above, the wavelet entropy values of the seven sections are displayed in Fig. 9(a). The wavelet entropy value in section $\mathrm{A}$ and $\mathrm{F}$ are obviously the largest because there were no striping defects. By selecting the signals from either section $\mathrm{A}$ or section $\mathrm{F}$ as $R E F$, we were able to use the correlation method to obtain the stripping size information from the other defects, as shown in Fig. 9(b). The stripping size, where $B<C<D<E$, was in accordance with the model design. The relative defect size was successfully identified.

The experimental results are in good agreement with the theoretical simulations, indicating that the combined signal processing technique of de-convolution, information entropy and cross-correlation can be used for qualitatively evaluating grouting quality.

As an example, a field measurement was conducted on a concrete structure, as shown in Fig. 10 (a). Using the methods discussed above, the bad grouting was obviously identified, as shown in Fig. 10 (b), by drilling examination in the concrete, the feasibility of the method is demonstrated. However, additional actual measurements need to be performed to improve the measurement accuracy and precision, particularly for relatively small defects.

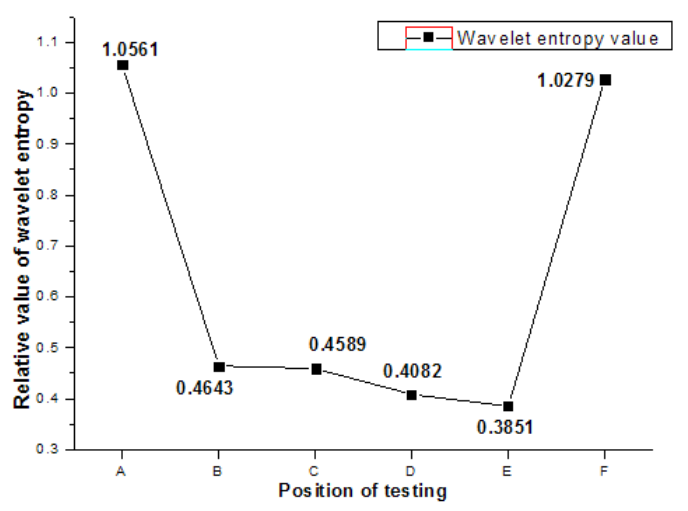

(a) Processing wavelet entropy

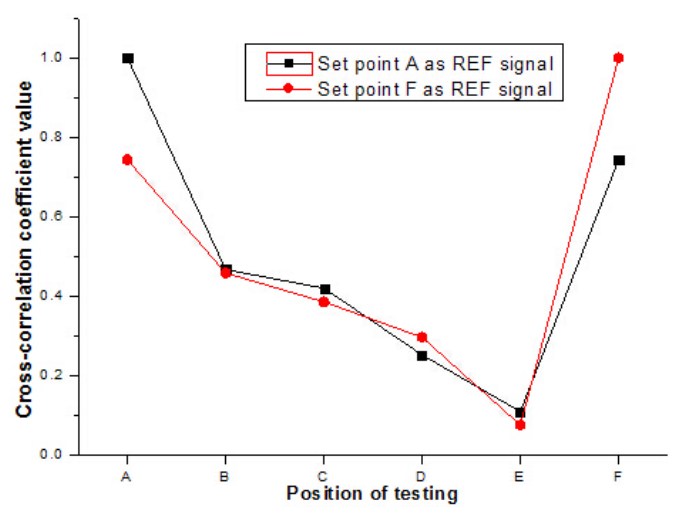

(b) Processing cross-correlation

Fig. 9 Processing the echo signal from the actual testing.

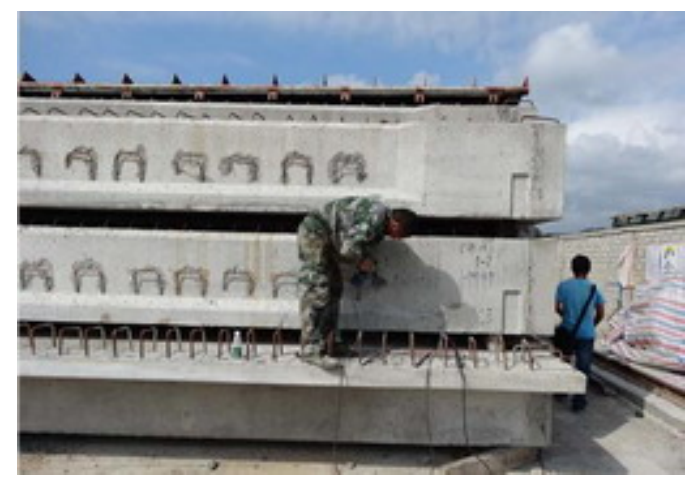

(a) Concrete structures with corrugated pipes

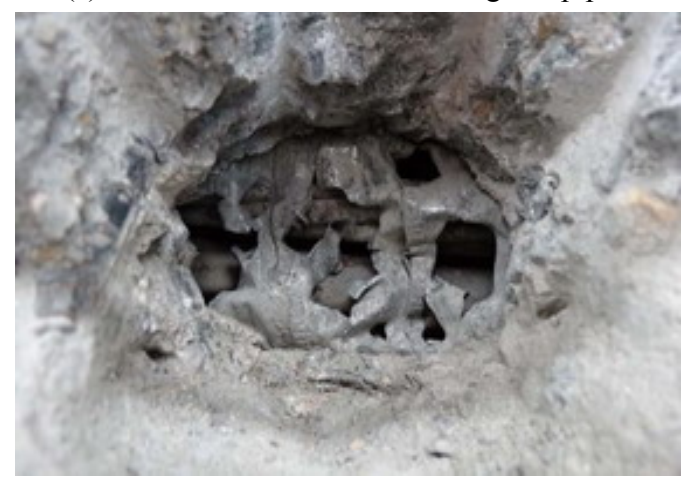

(b) The drill hole examination

Fig. 10 Field measurements by using method from this paper. 


\section{Conclusion}

In this paper, an application of the impact-echo technique and a combined signal processing method based on wavelet entropy and cross-correlation were presented for analyzing corrugated pipe grouting. Simulations and experiments were carried out to assess the effectiveness of the proposed NDT method. The simulation of sound field in the corrugated pipe demonstrated complex wave propagation characteristics that masked the grouting defects. The echo signals are first processed using de-convolution technology to extract the system signals. Using the wavelet entropy, the reference signal $(R E F)$ can be obtained by selecting the signal with the maximum entropy value. Then, the cross-correlation coefficients are used to evaluate the grouting quality. A concrete specimen containing some artificial defects (different stripping size) was used to test the theoretical predictions, and fairly good agreement was obtained. A field measurement was also conducted to demonstrate the feasibility of the method. In fact, the grouting quality detection is a very complicated problem, as a kind of exploration, the proposed method has its limitation and ought to combine other methods to finish synthetic judgment. Further work to combine other methods and determine quantitative criteria for different sized defects will be necessary to make this work more widely applicable.

\section{Acknowledgements}

This work were supported by National Natural Science Foundation of China (No.11274091, 11274092, 61302124) and the Funds for the Central Universities of Hohai University (Grant No. 2011B11014)

\section{References}

Beben, D., (2011). "Application of the interferometric radar for dynamic tests of corrugated steel plate (CSP) culvert." NDT \& E International, 44(5), 405-412.

Biatynicki-Birula, I. and Mycielski, J., (1975). "Uncer- tainty relations for information entropy in wave mechanics." Commun. Math. Phys, 44, 129-132.

Chaki, S. and Bourse, G., (2009). "Guided ultrasonic waves for non-destructive monitoring of the stress levels in prestressed steel strands." Ultrasonics, 49(2), 162-171.

Delvasto, S., Toro, E. F. Perdomo, F. and de Gutiérrez, R. M., (2010). "An appropriate vacuum technology for manufacture of corrugated fique fiber reinforced cementitious sheets." Construction and Building Materials, 24(2), 187-192.

Jaynes, E. T., (1982). "On the rationale of maximum-entropy method." Proceedings of the IEEE, 70(9), 939-952.Laasri, E. A., Akhouayri, E.-S., Agliz, D. and Atmani, A., (2014). "Automatic detection and picking of P-wave arrival in locally stationary noise using cross-correlation." Digital Signal Processing, 26, 87-100.

Lavrentyev, A. I. and Beals, J. T., (2000). "Ultrasonic measurement of the diffusion bond strength." Ultrasonics, 38(1-8), 513-516.

Liang, M.-T. and Su, P.-J., (2001). "Detection of the corrosion damage of rebar in concrete using impact-echo method." Cement and Concrete Research, 31(10), 1427-1436.

Lin, C.-C., Liu, P.-L. and Yeh, P.-L., (2009). “Application of empirical mode decomposition in the impact-echo test." NDT\&E International, 42(7), 589-598.

Shannon, C. E., (1948). "A mathematical theory of communication." Bell System Technical Journal: Boulogne-Billancourt, France, 379-423.

Perez-Canales, D., Alvarez-Ramirez, J. and JaureguiCorrea, J. C., Vela-Martinez, L. and Herrera-Ruiz, Gilberto., (2011). "Identification of dynamic instabilities in machining process using the approximate entropy method." International Journal of Machine Tools \& Manufacture, 51(6), 556-564.

Wan, X. and Wu, Z., (2013). "Sound source localization based on discrimination of cross-correlation functions." Applied Acoustics, 74(1), 28-37. 